\title{
The WASA Detector Facility at CELSIUS
}

\section{CELSIUS/WASA Collaboration}

Chr. Bargholtz ${ }^{\text {a }}$, M. Bashkanov ${ }^{\mathrm{b}}$, M. Berłowski $^{\mathrm{c}}$, A. Bondar $^{\mathrm{d}}$, D. Bogoslawsky ${ }^{\mathrm{e}}, \mathrm{W}$. Brodowski ${ }^{\mathrm{b}}$, J. Brudvik $^{\mathrm{f}}, \mathrm{H}$. Calén ${ }^{\mathrm{g}}$, F. Capellaro ${ }^{g}$, A. Chilingarov ${ }^{\mathrm{d}}$, H. Clement ${ }^{\mathrm{b}}$, J. Comfort ${ }^{\mathrm{h}}$, L. Demirörs ${ }^{i}$, C. Ekström ${ }^{j}$, K. Fransson ${ }^{g}$, C.-J. Fridén ${ }^{j}$, L. Gerén ${ }^{\mathrm{a}}$, M. Gornov k, V. Grebenev ${ }^{\mathrm{k}}$, J. Greiff ${ }^{\mathrm{i}}$, Y. Gurov ${ }^{\mathrm{k}}$,

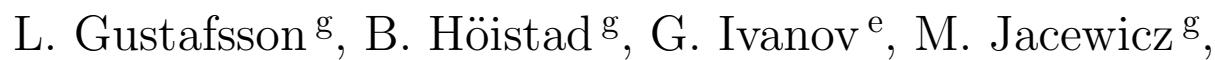
E. Jiganov ${ }^{\mathrm{e}}$, A. Johansson ${ }^{\mathrm{g}}$, T. Johansson ${ }^{\mathrm{g}}$, S. Keleta ${ }^{\mathrm{g}}$, O. Khakimova ${ }^{\mathrm{b}}$, A. Khoukaz ${ }^{\ell}, \mathrm{K}$. Kilian ${ }^{\mathrm{m}}$, N. Kimura ${ }^{\mathrm{n}}$, I. Koch ${ }^{g}$, G. Kolachev ${ }^{d}$, F. Kren ${ }^{\text {b }}$, S. Kullander ${ }^{\mathrm{g}}$, A. Kupść ${ }^{\mathrm{g}}$, A. Kuzmin ${ }^{d}$, A. Kuznetsov ${ }^{\mathrm{e}}, \mathrm{K}$. Lindberg ${ }^{\mathrm{a}}$, P. Marciniewski ${ }^{\mathrm{g}}$, R. Meier ${ }^{\mathrm{b}}$, O. Messner $^{\mathrm{b}}$, B. Morosov ${ }^{\mathrm{e}}$, A. Nawrot $^{\mathrm{c}}$, B.M.K. Nefkens ${ }^{\mathrm{f}}$, G. Norman ${ }^{\mathrm{j}}$, W. Oelert ${ }^{\mathrm{m}}$, C. Pauly $^{\mathrm{i}}$, H. Pettersson ${ }^{\mathrm{g}}$, A. Pivovarov ${ }^{\mathrm{d}}$, J. Pätzold ${ }^{\mathrm{b}}$, Y. Petukhov ${ }^{\mathrm{e}}$, A. Povtorejko $^{\mathrm{e}}$, D. Reistad ${ }^{\mathrm{j}}$, R.J.M.Y. Ruber ${ }^{\mathrm{g}}$, S. Sandukovsky ${ }^{\mathrm{e}}$, K. Schönning ${ }^{\mathrm{g}}$, W. Scobel ${ }^{\mathrm{i}}$, T. Sefzick ${ }^{\mathrm{m}}$, R. Shafigullin ${ }^{k}$, B. Shwartz ${ }^{\text {d, V. Sidorov }}{ }^{\text {d }}$ T. Skorodko ${ }^{\text {, }}$, V. Sopov ${ }^{\circ}$, A. Starostin ${ }^{\text {, }}$, J. Stepaniak ${ }^{c}$, A. Sukhanov ${ }^{\text {, }}$,

A. Sukhanov ${ }^{\mathrm{e}}, \mathrm{V}$. Tchernyshev ${ }^{\mathrm{O}}$, P.-E. Tegnér ${ }^{\mathrm{a}}$, P. Thörngren Engblom ${ }^{g}$, V. Tikhomirov ${ }^{\mathrm{e}}, \mathrm{H}$. Toki ${ }^{\mathrm{p}}$, A. Turowiecki ${ }^{\mathrm{q}}$, G.J. Wagner ${ }^{\mathrm{b}}$, U. Wiedner ${ }^{\mathrm{g}}, \mathrm{Z}$. Wilhelmi ${ }^{\mathrm{q}}$, M. Wolke ${ }^{\mathrm{m}}$, A. Yamamoto ${ }^{\mathrm{n}}, \mathrm{H}$. Yamaoka ${ }^{\mathrm{n}}$, J. Zabierowski ${ }^{\mathrm{c}}$, I. Zartova ${ }^{a}$, J. Złomańczuk ${ }^{\mathrm{g}}$ a Stockholm University, Stockholm, Sweden

b Physikalisches Institut der Universität Tübingen, Tübingen, Germany

${ }^{\mathrm{c}}$ Soltan Institute of Nuclear Studies, Warsaw and Lodz, Poland

d Budker Institute of Nuclear Physics, Novosibirsk, Russia

e Joint Institute for Nuclear Research, Dubna, Russia

${ }^{\mathrm{f}}$ University of California Los Angeles, Los Angeles, California, USA

g Uppsala University, Uppsala, Sweden

${ }^{\mathrm{h}}$ Arizona State University, Tempe, USA 


\author{
${ }^{\mathrm{i}}$ Institut für Experimentalphysik der Universität Hamburg, Hamburg, Germany \\ j The Svedberg Laboratory, Uppsala, Sweden \\ ${ }^{\mathrm{k}}$ Moscow Engineering Physics Institute, Moscow, Russia \\ ${ }^{\ell}$ Münster University, Münster, Germany \\ ${ }^{\mathrm{m}}$ Institut für Kernphysik, Forschungszentrum Jülich, Jülich, Germany \\ ${ }^{\mathrm{n}}$ High Energy Accelerator Research Organization, Tsukuba, Japan \\ ${ }^{\circ}$ Institute of Theoretical and Experimental Physics, Moscow, Russia \\ ${ }^{\mathrm{p}}$ Research Centre for Nuclear Physics, Osaka, Japan \\ ${ }^{\mathrm{q}}$ Institute of Experimental Physics of Warsaw University, Warsaw, Poland
}

\begin{abstract}
The WASA $4 \pi$ multidetector system, aimed at investigating light meson production in light ion collisions and $\eta$ meson rare decays at the CELSIUS storage ring in Uppsala is presented. A detailed description of the design, together with the anticipated and achieved performance parameters are given.
\end{abstract}

Key words:

PACS: 29.40.-n, 29.40.Mc, 29.40.Cs, 13.20.-v, 14.40.Aq

\title{
1 Introduction
}

The $4 \pi$ detector facility WASA 1 was designed for studies of production and decays of light mesons in an internal-target experiment at the CELSIUS accelerator and cooler storage ring (1). The highest beam-proton kinetic energy reachable at CELSIUS was $1.5 \mathrm{GeV}$ and protons of energies up to $500 \mathrm{MeV}$ could be electron cooled. Single and multipion production in proton-proton, proton-deuteron and deuteron-deuteron interactions were studied over a wide range of beam energies. The available beam energies also allowed production of eta mesons in proton-proton and proton-deuteron reactions and production of omega mesons in proton-deuteron reactions.

The WASA project required some special technical developments e.g. a very thin-walled superconducting solenoid and a hydrogen pellet target. The pellet concept is crucial to achieve a close to $4 \pi$ detection acceptance in this internaltarget storage ring experiment.

${ }^{1}$ CELSIUS/WASA Homepage, http://www.tsl.uu.se/wasa 
The WASA detector was installed at CELSIUS in 1999 and from 2002 it was fully equipped with read-out electronics. The WASA experiments were assigned in total about 6500 hours of beam time until June 2005 when CELSIUS was closed down. WASA was then moved to COSY at Forschungzentrum Jülich, Germany, where it successfully started operation again in the autumn of 2006.

In the CELSIUS/WASA experiment several production reactions were studied e.g.: $p d \rightarrow{ }^{3} \mathrm{He} \pi \pi(2), p p \rightarrow p p 3 \pi(\underline{3}), p p \rightarrow p p \pi^{0}(\underline{4}), p d \rightarrow{ }^{3} \mathrm{He} \omega, d d \rightarrow{ }^{4} \mathrm{He} \pi \pi$. Some $\eta$ decays e.g. $\eta \rightarrow 3 \pi^{0}(\underline{5}), \eta \rightarrow \pi^{+} \pi^{-} \mathrm{e}^{+} \mathrm{e}^{-}(\underline{6})$ and $\eta \rightarrow \mathrm{e}^{+} \mathrm{e}^{-} \gamma(7)$ were studied in $p d \rightarrow{ }^{3} \mathrm{He} \eta$ and $p p \rightarrow p p \eta$ reactions. New upper limits for the $\eta \rightarrow \mathrm{e}^{+} \mathrm{e}^{-} \mathrm{e}^{+} \mathrm{e}^{-}$and $\eta \rightarrow \mu^{+} \mu^{-} \mu^{+} \mu^{-}$decay branching ratios (7) have been obtained.

\section{The WASA detector}

The WASA setup at CELSIUS, shown in the form of a CAD drawing in Fig. 1, can be divided into four major parts: the pellet-target, the forward detector (FD), the zero-degree spectrometer (ZD) and the central detector (CD).

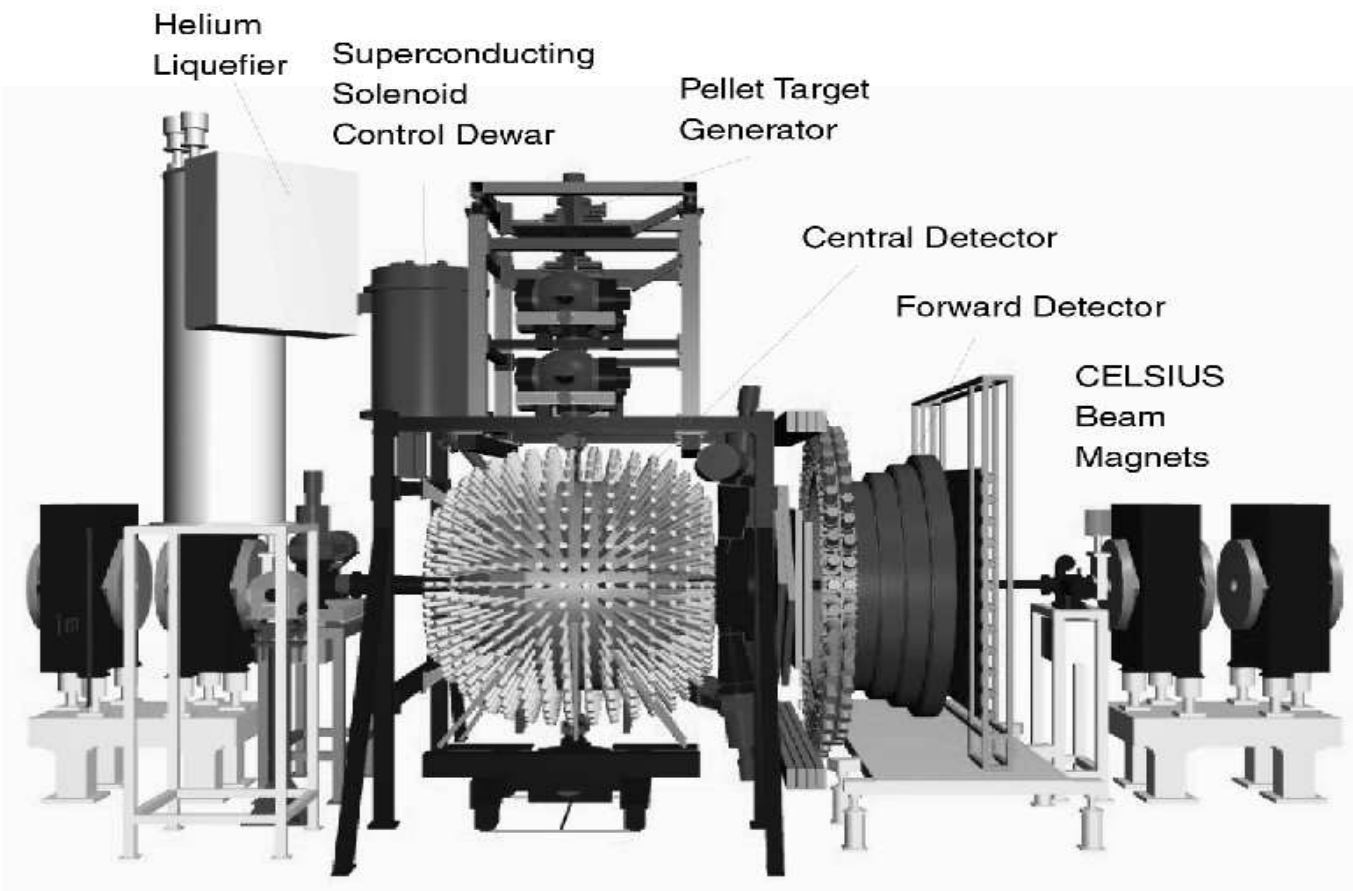

Fig. 1. CAD view of the WASA Detector Facility. The zero-degree spectrometer is located further downstream to the right.

The pellet-target system is integrated in the setup and it provides small spheres of frozen hydrogen or deuterium as internal targets. This allows high 
luminosity and high detection coverage for meson decay products like photons, electrons and charged pions.

The FD and ZD measure charged target-recoil particles and scattered projectiles. The FD consists of eleven planes of plastic scintillators and of proportional counter drift tubes. The ZD contains detectors placed in the CELSIUS beam magnets and provides information on forward going particles, that would otherwise be lost in the beam pipe. This was exploited e.g. for the identification of $\mathrm{pd} \rightarrow{ }^{3} \mathrm{He} \eta$ events measured close to the kinematic threshold. The CD was designed for measurements of the meson decay products and consists of an electromagnetic calorimeter of $\mathrm{CsI}(\mathrm{Na})$ crystals surrounding a superconducting solenoid. Inside of the solenoid a cylindrical chamber of drift tubes and a barrel of plastic scintillators are placed. A vertical cross section of the WASA detector is shown in Fig. 2.

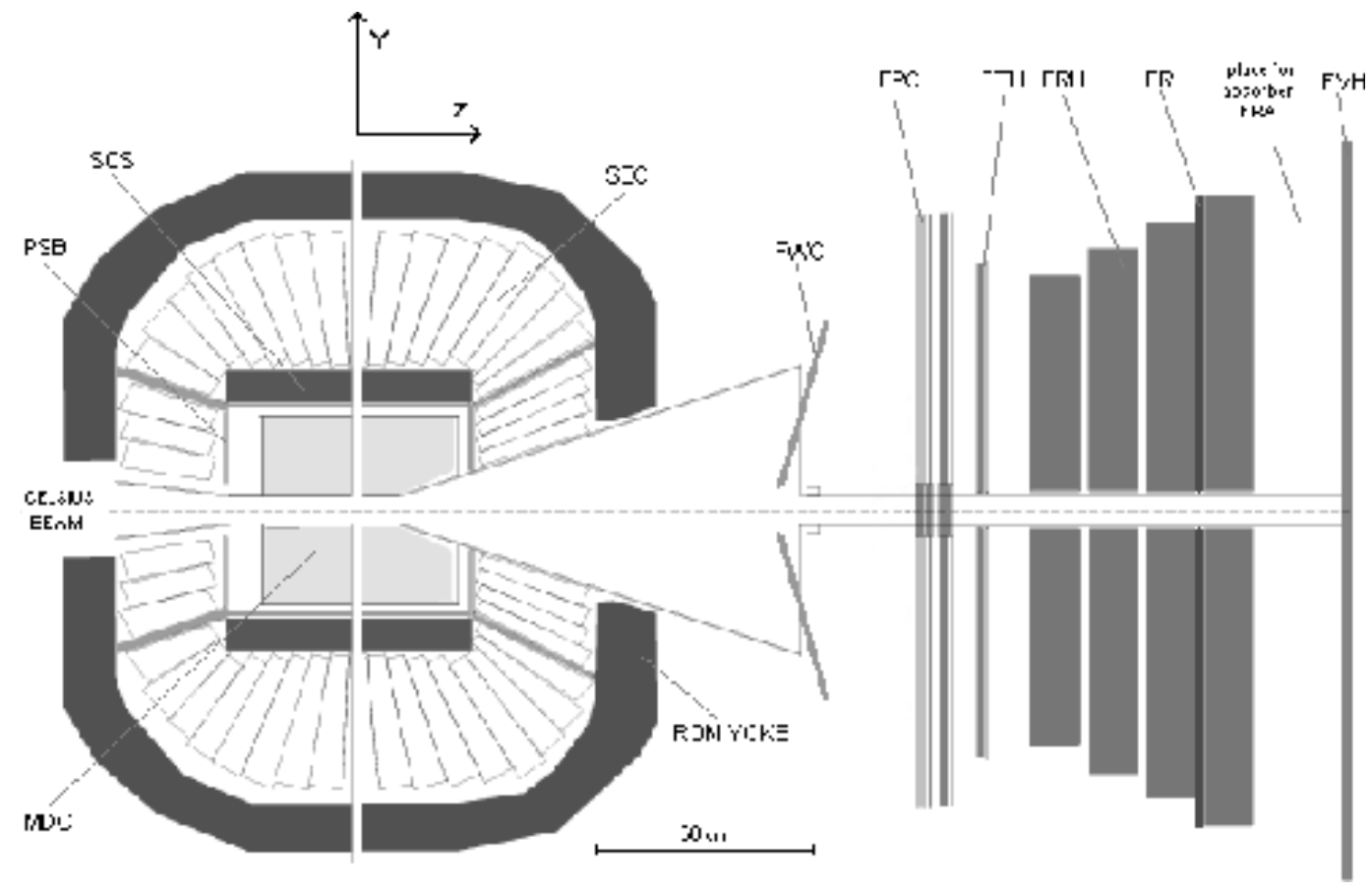

Fig. 2. Cross section of the WASA detector. The central detector built around the interaction point (at the left) is surrounded by an iron yoke. The layers of the forward detector are visible on the right-hand side. The individual components are described in the text.

\subsection{Pellet target}

The pellet target system was a unique development for the CELSIUS/WASA experiments $(8 ; 9)$. The main components of the system are shown in Fig. 3. The heart of the setup is the pellet generator where a jet of liquid hydrogen is broken up into droplets with a diameter of about $35 \mu \mathrm{m}$ by a vibrating nozzle. 


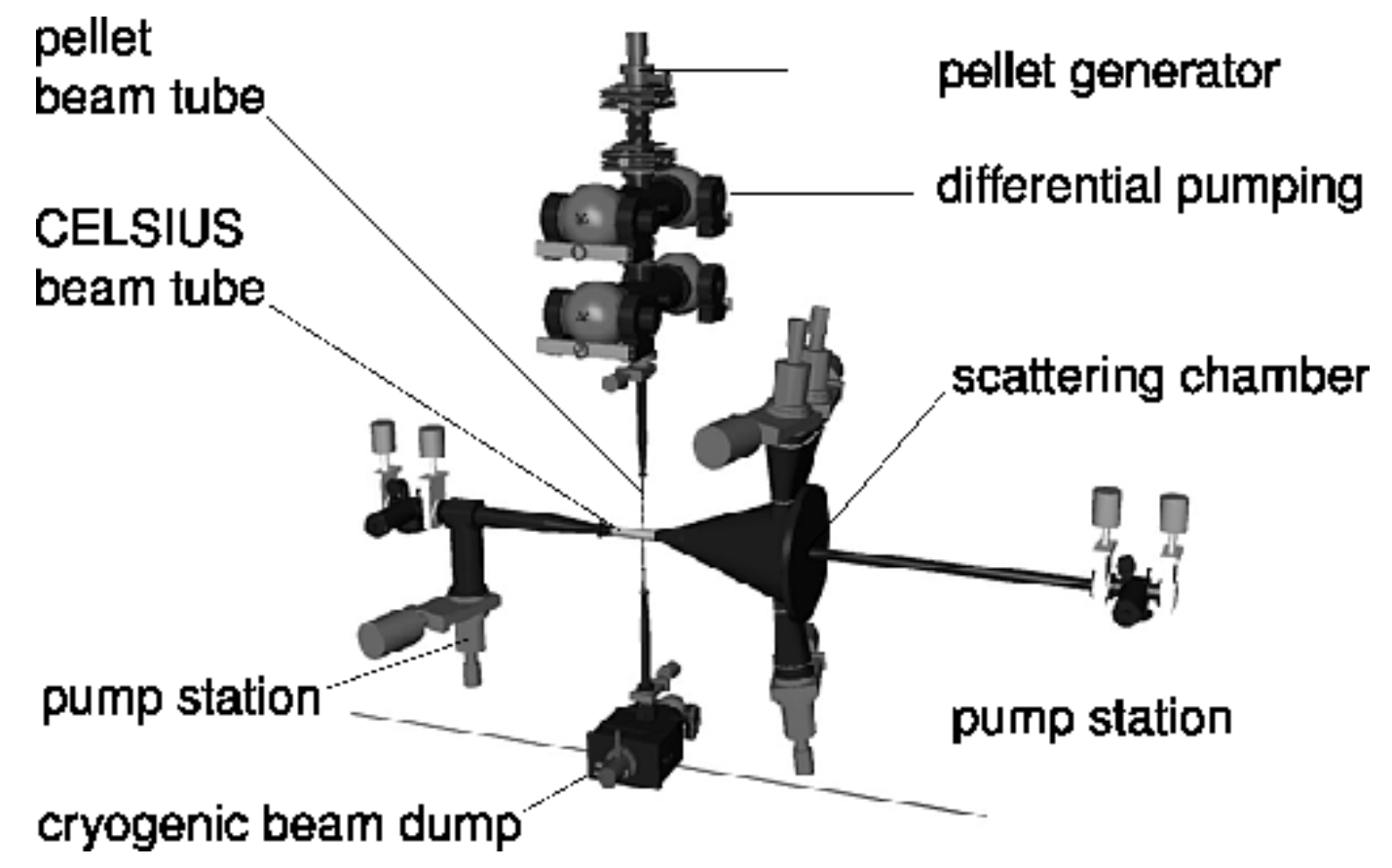

Fig. 3. Layout of the pellet target system.

The droplets freeze by evaporation in a droplet chamber and form a beam of pellets that pass a $7 \mathrm{~cm}$ long vacuum-injection capillary. After collimation, the pellets are directed through a thin $2 \mathrm{~m}$ long pipe into the scattering chamber and further down to a pellet beam dump. The inner diameter of the pipe is $5 \mathrm{~mm}$ at the entrance to the scattering chamber. This arrangement provides the necessary space to put the $4 \pi$ detection system around the interaction region. Pellet target thicknesses of up to $3 \cdot 10^{15}$ atoms $/ \mathrm{cm}^{2}$ gave acceptable half-lives of the circulating ion beam as well as acceptable vacuum conditions. Some of the parameters of the pellet target are listed in table 1. The pellet target system was regularly operated with pellets of normal hydrogen or deuterium.

Table 1

\begin{tabular}{lc}
\hline Pellet diameter $[\mu \mathrm{m}]$ & $25-35$ \\
Pellet frequency $[\mathrm{kHz}]$ & $5-12$ \\
Pellet - pellet distance $[\mathrm{mm}]$ & $9-20$ \\
Effective target thickness $\left[\right.$ at.s $\left./ \mathrm{cm}^{2}\right]$ & $>10^{15}$ \\
Beam diameter $[\mathrm{mm}]$ & $2-4$ \\
\hline
\end{tabular}

Performance of the pellet target system. 


\subsection{Forward Detector}

The FD was designed mainly for detection and identification of scattered projectiles and charged recoil particles like protons, deuterons and He nuclei in $\pi$ and $\eta$ production reactions. Also neutrons and charged pions can be measured. All FD plastic scintillators may supply information for the first level trigger logic. Most of the components of this part of the setup were used in a previous experiment at CELSIUS, PROMICE-WASA, which is described in more detail in ref. (10). A summary of the most important features of the forward detector is given in table 2. In this section the individual components are described in some detail with emphasis on the extensions with respect to the PROMICE-WASA setup .

Total number of scintillator elements

Scattering angle coverage [degrees]

Scattering angle resolution [degrees]

Amount of sensitive material $\left[\mathrm{g} / \mathrm{cm}^{2}\right]$

[radiation lengths]

[nuclear interaction lengths]

Thickness of vacuum window (st. steel) [mm]

Maximum kinetic energy $\left(T_{\text {stop }}\right)$ for stopping:

$$
\pi^{ \pm} / \text {proton/deuteron/alpha }
$$

Time resolution [ns]
280

$3-17$

0.2

50

$\approx 1$

$\approx 0.6$

$\approx 0.4$

Energy resolution for:

stopped particles

particles with $T_{\text {stop }}<T<2 T_{\text {stop }}$

$\approx 3 \%$

$4-8 \%$

Particle identification

Table 2

Some features of the Forward Detector.

The Forward Window Counter (FWC) is the first detector layer in the FD (along the beam direction) and consists of 12 plastic scintillators of $5 \mathrm{~mm}$ thickness (Fig. 4). It is mounted tightly on the paraboloidal stainless steel vacuum window. Therefore, the elements are inclined with approximately $19^{\circ}$ with respect to the plane perpendicular to the beam direction. The FWC signals are used in the first level trigger logic to reduce the background caused by particles scattered in the downstream beam pipe and in the flange at the entrance to the FD. The signals are also used to select He ejectiles at the trigger level. 


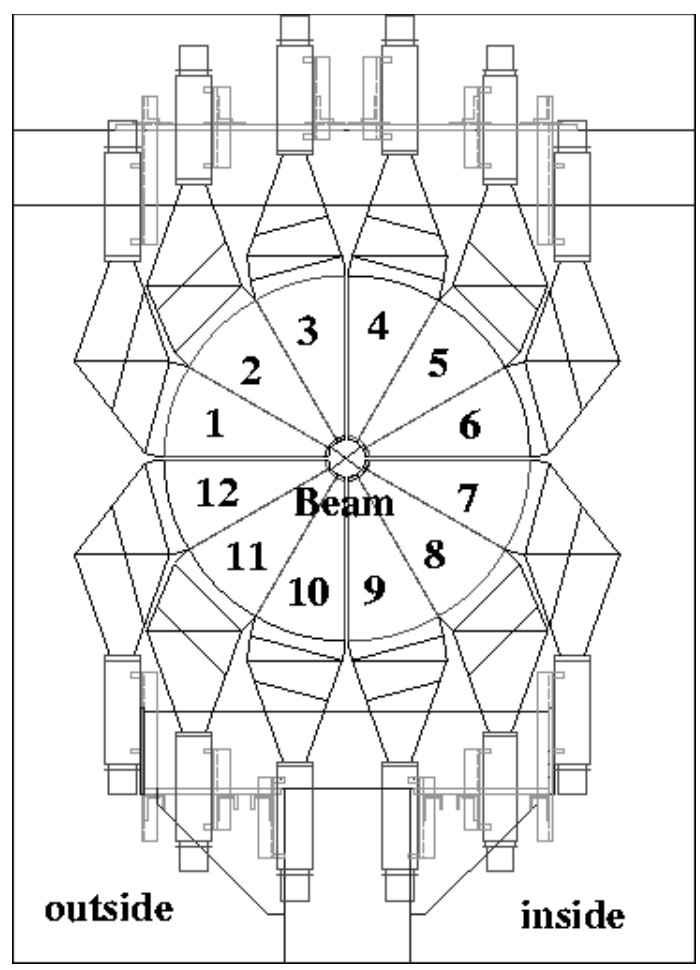

Fig. 4. Schematic view of the FWC.

Downstream the FWC, there is a tracking device: the Forward Proportional Chambers (FPC). It is composed of four modules, each with four staggered layers of 122 proportional drift tubes (so called straws) of $8 \mathrm{~mm}$ diameter. The straws, made of $26 \mu \mathrm{m}$ thick aluminized Mylar and with a central sense wire of stainless steel with $35 \mu \mathrm{m}$ diameter, are operated with a 50/50 $\mathrm{Ar} / \mathrm{CO}_{2}$ gas mixture. The modules are rotated by $45^{\circ}$ with respect to each other (in the plane perpendicular to the beam axis). They are used for accurate reconstruction of track coordinates and provide precise angular information of the particles originating from the target region. The FPC is described in detail in ref. (10) and in the Ph.D. thesis of J. Dyring (11).

The Forward Trigger Hodoscope (FTH), consisting of three layers of $5 \mathrm{~mm}$ thick plastic scintillators, is placed next to the FPC. There are 24 Archimedean spiral-shaped elements in the first two planes and 48 radial elements in the third. The special geometry, combining all three layers, results in a pixel structure, which is useful for resolving multi-hit ambiguities (12). The FTH was designed to provide scattering angle information in the first level trigger logic.

Behind the FTH, the four layers of the Forward Range Hodoscope (FRH) are positioned. Each plane is made of 24 plastic scintillator modules each of $11 \mathrm{~cm}$ thickness. The FRH, together with FTH, is used for energy determination of charged particles and for particle identification by $\Delta E-E$ technique. Fig. 5 shows how protons, deuterons and He nuclei can be identified. 


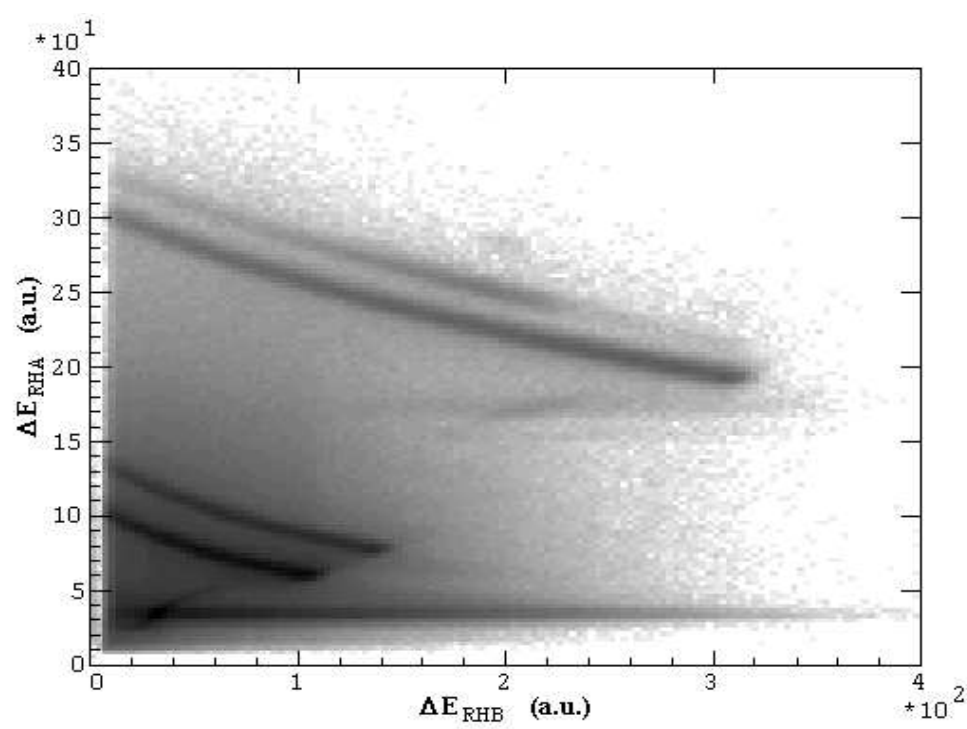

Fig. 5. An example of $\Delta E-\Delta E$ information from a deuteron-deuteron run at $1012 \mathrm{MeV}$ used for particle identification in the forward detector. Energy deposited in the first layer of FRH is plotted versus the energy deposited in the second layer of FRH. The upper two bands are from ${ }^{4} \mathrm{He}$ and ${ }^{3} \mathrm{He}$ nuclei and in the lower part of the plot there are two bands from deuterons and protons.

The identity and initial kinetic energy of a charged particle is reconstructed from the pattern of deposited energy in the different detector planes. Even for particles that are stopped in a detector, the total deposited energy is different from the initial kinetic energy. This is because some of the energy is lost in inactive material between the detectors elements, in the scattering chamber window, etc. The variation of the deposited energy is strong enough to be useful for energy reconstruction also for high energy particles not stopped in the detector material. For protons, this can be used in the kinetic energy range $300 \mathrm{MeV}$ to $800 \mathrm{MeV}$ and for deuterons in a similar energy range starting from $400 \mathrm{MeV}$. Identification of punch-through particles can be done using either the forward veto hodoscope or the $\Delta E$ information from the last $\mathrm{FRH}$ planes. More details on FTH, FRH and the method for particle energy reconstruction are given in ref. (10).

Between the third and fourth layers of the FRH there are two interleaving layers of $5 \mathrm{~mm}$ thick plastic scintillator bars with one-sided readout (Fig. 6), the Forward Range Interleaving Hodoscope (FRI). Each layer has 32 bars, oriented horizontally in one and vertically in the other. The main purpose of this addition to the FRH is to provide a two-dimensional position sensitivity inside the FRH necessary for measurement of scattering angles for neutrons. The probability that a neutron of a few hundred MeV kinetic energy will interact in the FRH is around $35 \%$. The FRI can help to reconstruct outgoing protons from the nuclear interaction and to discriminate against background tracks due to secondary interactions in the beam pipe and other structural 
material. More information about the design and performance of the FRI can be found in references $(13 ; 14)$.

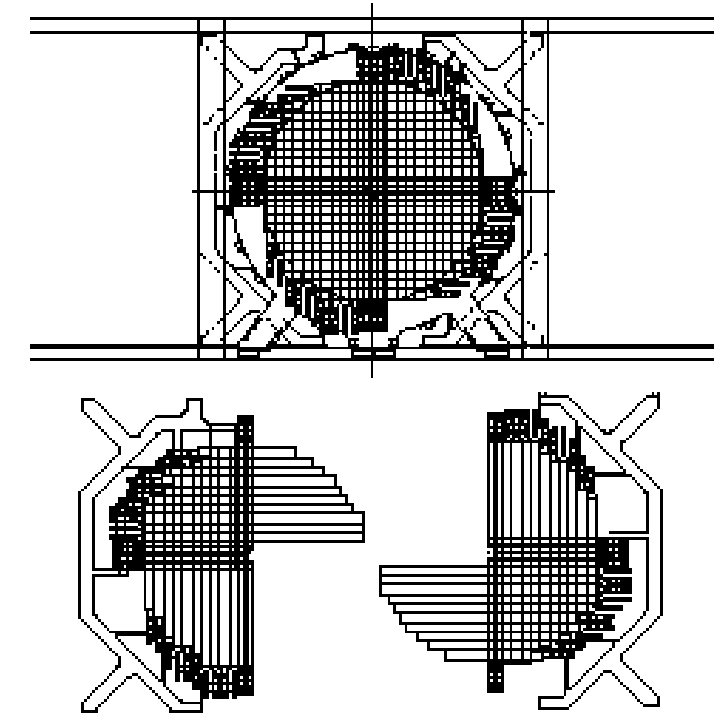

Fig. 6. Schematic view of the FRI detector (upper picture) and its two planes with orthogonally oriented scintillator bars (lower picture).

The last detector layer of the FD is a wall of plastic scintillators (Fig. 7), the Forward Veto Hodoscope (FVH). It consists of 12 horizontal plastic scintillator bars, equipped with photomultipliers on both ends. The hit position along a bar may be reconstructed from signal time information with a resolution of about $5 \mathrm{~cm}(\sigma)$. In the first level trigger the signals are used for rejection (or selection) of particles punching through the FRH.

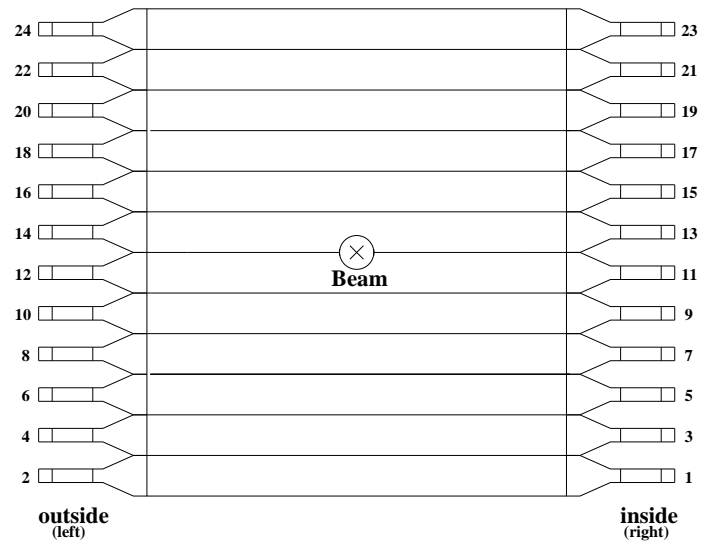

Fig. 7. Schematic view of the Forward Veto Hodoscope.

Optionally, a passive absorber layer (FRA) made of iron can be introduced between the last layer of the FRH and the FVH. The thickness of the absorber can be chosen from $5 \mathrm{~mm}$ up to $100 \mathrm{~mm}$. Immediately in front of and behind the iron are placed $10 \mathrm{~mm}$ thick plexi-glass sheets. The absorber has been used for stopping the protons from the $p p \rightarrow p p \eta$ reaction at a beam proton energy around $1360 \mathrm{MeV}$. In this case the faster protons from elastic scattering and 
from pion production penetrate the FRA and induce signals in the FVH which can be used for veto purposes in the first level trigger.

\subsection{Zero-Degree spectrometer}

The ZD uses the CELSIUS dipole magnets for filtering out low momentum reaction products escaping into the beam pipe (figure 8). The reaction products could be detected at different port positions of the vacuum-chamber in the dipole magnets (about $6.6 \mathrm{~m}$ away from the target). A range of magnetic rigidities relative to that of the circulating beam $(B \rho)_{\text {rel }}$ of 0.3 to 0.8 could be covered. The acceptance depends critically on the size of the detectors and on the settings of the quadrupole magnets. The maximum geometric acceptance, $0.7 \mathrm{msr}$, is reached at a relative rigidity of 0.65 . A stainless steel window of $100 \mu \mathrm{m}$ thickness at the port position allowed the use of external setups of detectors. Setups of 6 layers of $12 \mathrm{CsI}(\mathrm{Tl})$ crystals, some front Si-strip detectors and plastic scintillators were prepared for this purpose.

The main detector setup was a telescope of silicon and germanium detectors cooled to liquid-nitrogen temperature installed inside of the vacuum-chamber. This was used for very clean tagging of $\eta$ production in $p d \rightarrow{ }^{3} \mathrm{He} \eta$ reactions near the threshold. The telescope comprised two thin silicon and two thicker germanium detectors. The silicon detectors installed in front of the germanium ones are used as transmission $(\Delta E)$ elements for particle identification. The ${ }^{3} \mathrm{He}$ ions from the $p d \rightarrow{ }^{3} \mathrm{He} \eta$ reaction are stopped in the thicker germanium detectors. ${ }^{3} \mathrm{He}$ ions with an energy of up to approximately $400 \mathrm{MeV}$ were fully stopped in the detector telescope (Fig. 9). The energy resolution (FWHM) of the telescope under normal CELSIUS conditions for ${ }^{3} \mathrm{He}$ ions with an energy of approximately $300 \mathrm{MeV}$ is estimated to be about $1.5 \mathrm{MeV}$. A detailed description of this detector system and its performance can be found in ref. (15).

\subsection{Central Detector}

The CD is built around the interaction point and was designed mainly for detection and identification of the decay products of $\pi^{0}$ and $\eta$ mesons: photons, electrons and charged pions. It consists of an inner drift chamber (MDC), thin plastic scintillators in a cylinder geometry (PSB), a solenoid (SCS) providing a magnetic field for momentum measurements and a CsI calorimeter (SEC). The amount of structural material is kept minimal to reduce the disturbances on the particles. The beam pipe is made of $1.2 \mathrm{~mm}$ thick beryllium and the total thickness of the solenoid corresponds to 0.18 radiation lengths only. The placement of the solenoid inside of the calorimeter allows the use of photomultipliers for readout. 


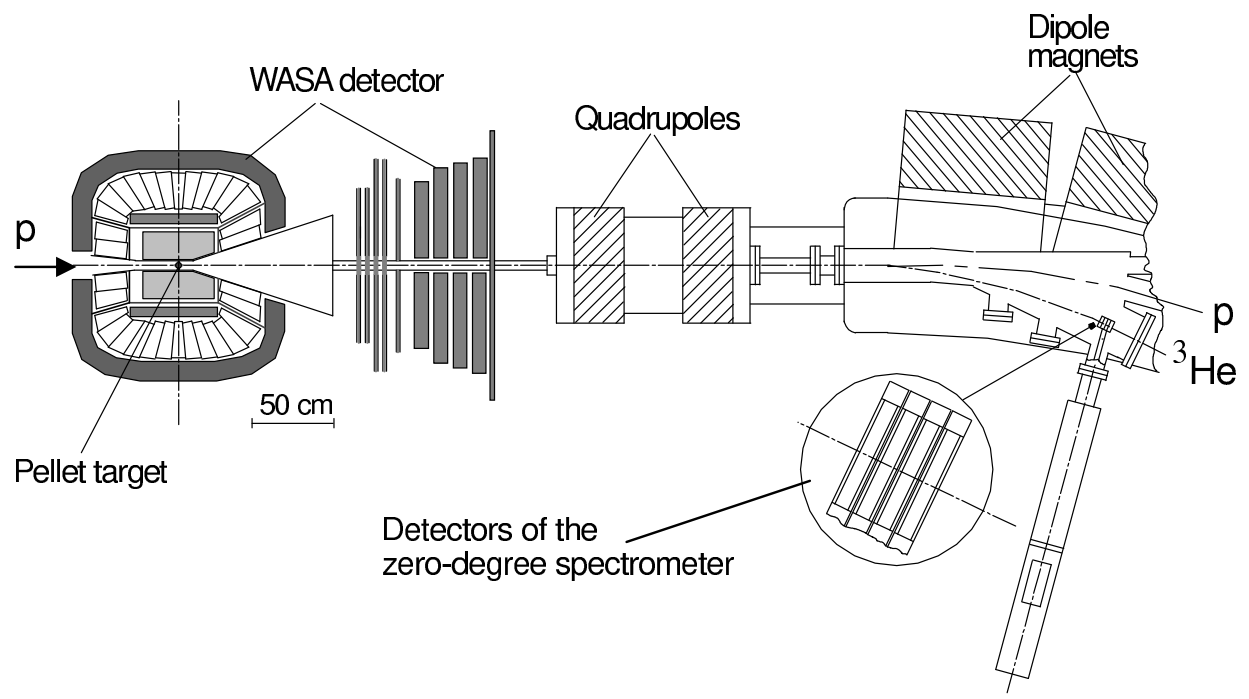

Fig. 8. Drawing of CELSIUS dipoles downstream the WASA detector with the zero-degree detector installation.

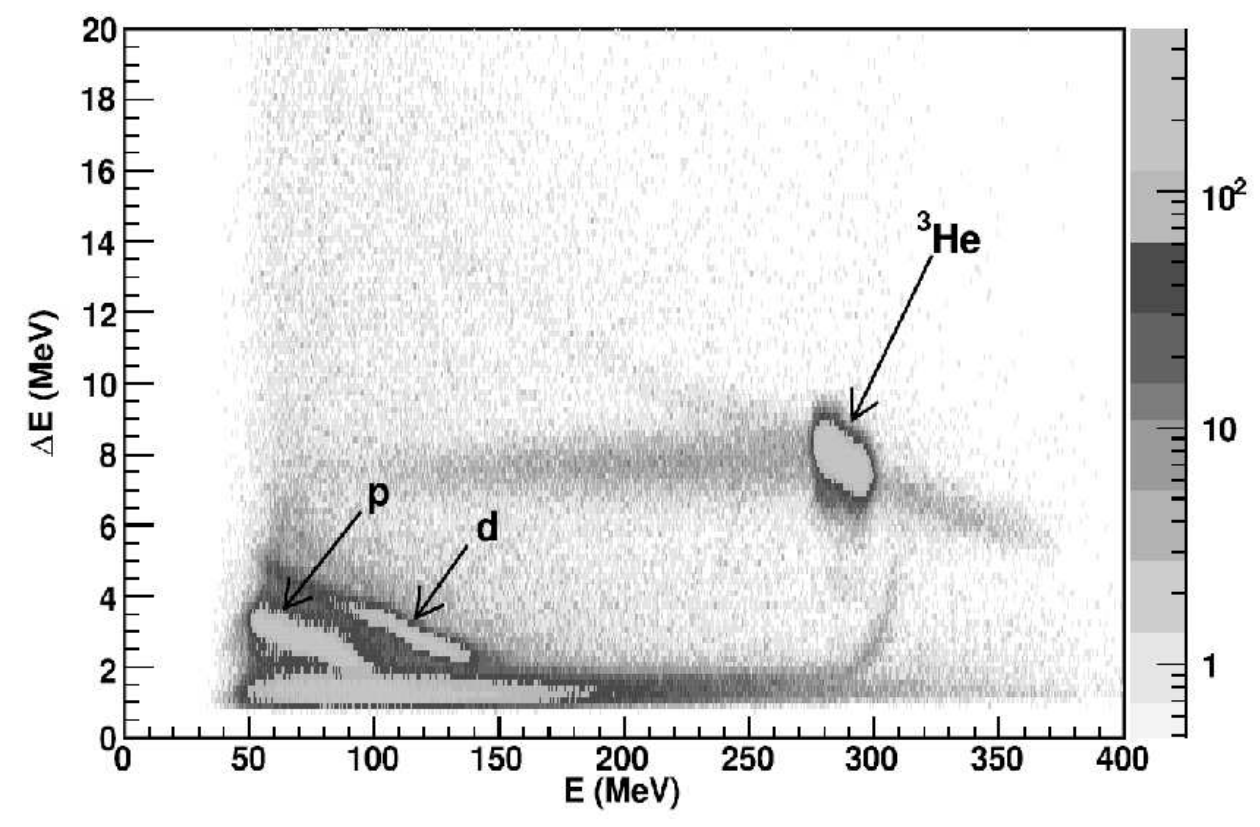

Fig. 9. An example of particle identification using $\Delta E-E$ information from the zero-degree spectrometer for a raw data sample of $p d$ interactions close to $p d \rightarrow{ }^{3} \mathrm{He} \eta$ threshold.

The main requirements for the design were the following:

- to handle high particle fluxes at luminosities around $10^{32} \mathrm{~cm}^{-2} \mathrm{~s}^{-1}$.

- to measure photons with energies from a few $\mathrm{MeV}$ up to $800 \mathrm{MeV}$.

- to measure, in a magnetic field of about $1 \mathrm{~T}$, the momenta of electrons and positrons in the range $20 \mathrm{MeV} / \mathrm{c}$ to $600 \mathrm{MeV} / \mathrm{c}$ with an accuracy $\sigma(p) / p \approx 2 \%$. 
The momenta of heavier charged particles can also be measured in a similar momentum range but with lower accuracy. For pions and muons an accuracy $\sigma(p) / p \approx 4 \%$ (for $p 100 \mathrm{MeV} / \mathrm{c}$ to $600 \mathrm{MeV} / \mathrm{c}$ ) can be obtained and for protons a $\sigma(p) / p \approx 6 \%$ (for $p 200 \mathrm{MeV} / \mathrm{c}$ to $800 \mathrm{MeV} / \mathrm{c}$ ).

The main components of the Central Detector, shown in Fig. 2, are presented below in some detail.

\subsubsection{The Superconducting Solenoid - (SCS)}

The SCS provides an axial magnetic field for the momentum measurements of the tracks measured by the MDC. It also protects the CD from low-energy delta electrons produced in the interactions of beam particles with the pellets. The wall thickness of the SCS is minimized in order to allow high accuracy of the energy measurements in the calorimeter. The return path for the magnetic flux is provided by a yoke made out of 5 tons of soft iron with low carbon content. The yoke shields the readout electronics from the magnetic field and also serves as support for the calorimeter crystals. The main parameters of the SCS are given in table 3 ,

\begin{tabular}{lr} 
Superconducting coil & \\
Inner/outer radius $[\mathrm{mm}]$ & $267.8 / 288.8$ \\
Superconductor (stabilizer) & $\mathrm{NbTi} / \mathrm{Cu}$ (pure Al) \\
Total winding length $[\mathrm{mm}]$ & 465 \\
Maximum central magnetic flux density, $\mathrm{B}_{c}[\mathrm{~T}]$ & 1.3 \\
Field uniformity in the MDC $[\mathrm{T}]$ & $1.22 \pm 0.25$ \\
Cooling (Liquid He) $[\mathrm{K}]$ & 4.5 \\
Cryostat & $\mathrm{Aluminium}$ \\
Material & $245 / 325$ \\
Inner / outer radius $[\mathrm{mm}]$ & 555 \\
Overall length $[\mathrm{mm}]$ & $\mathbf{0 . 1 8}$ \\
SCS wall thickness (coil+cryostat) $[\mathrm{radl}]$ & \\
\hline
\end{tabular}

Table 3

Main parameters of the superconducting coil and its cryostat.

In order to map the magnetic field inside the volume enclosed by the SCS, the magnetic field strength inside the MDC was measured with Hall probes and, in addition, the field distribution was calculated with simulation programs. The calculated values were fitted to the measured ones with an error of $\pm 1 \%$ of $\mathrm{B}_{\text {total }}$. The SCS is described in detail in ref. (16) and in the Ph.D. thesis of R. Ruber (17). 


\subsubsection{The Mini Drift Chamber - $(M D C)$}

The MDC is placed around the beam pipe and is used for determination of particle momenta and reaction vertex. It is a cylindrical chamber covering scattering angles from $24^{\circ}$ to $159^{\circ}$. For large angle scattered protons from elastic proton-proton scattering, a vertex resolution $(\sigma)$ of $0.2 \mathrm{~mm}$ perpendicular and $3 \mathrm{~mm}$ along the beam axis can be reached. A detailed description of the MDC can be found in the Ph.D. thesis of M. Jacewicz (18).

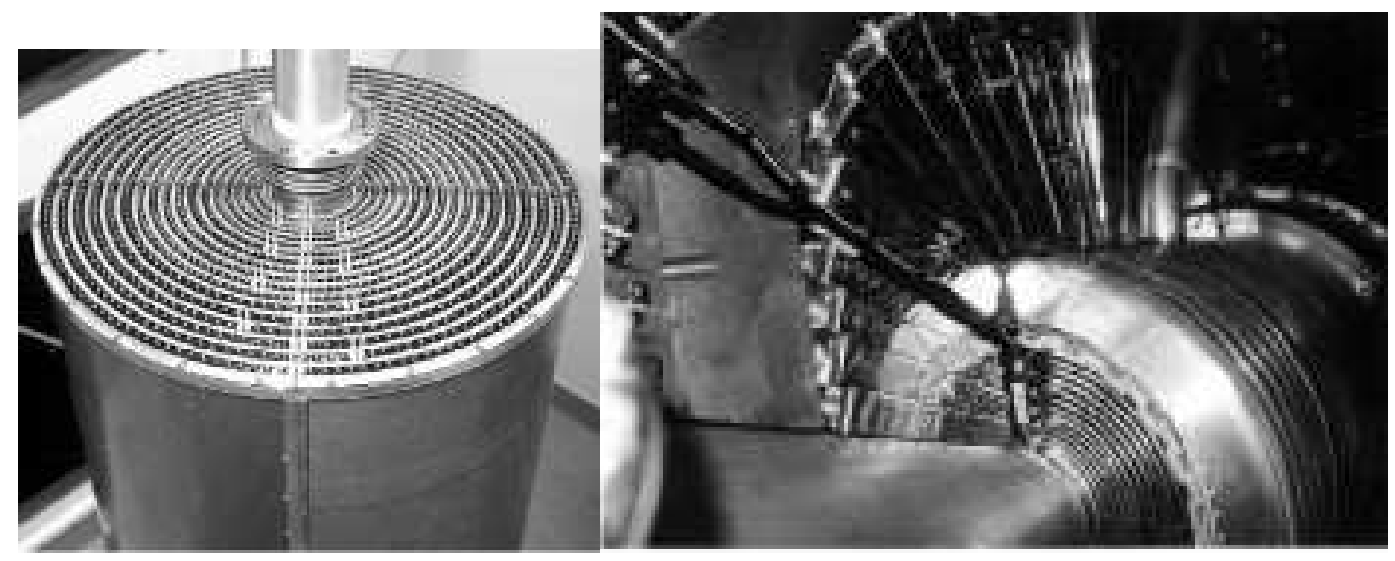

Fig. 10. (Left) The fully assembled MDC inside the Al-Be cylinder. (Right) The MDC surrounded by PSB elements and the SCS cryostat.

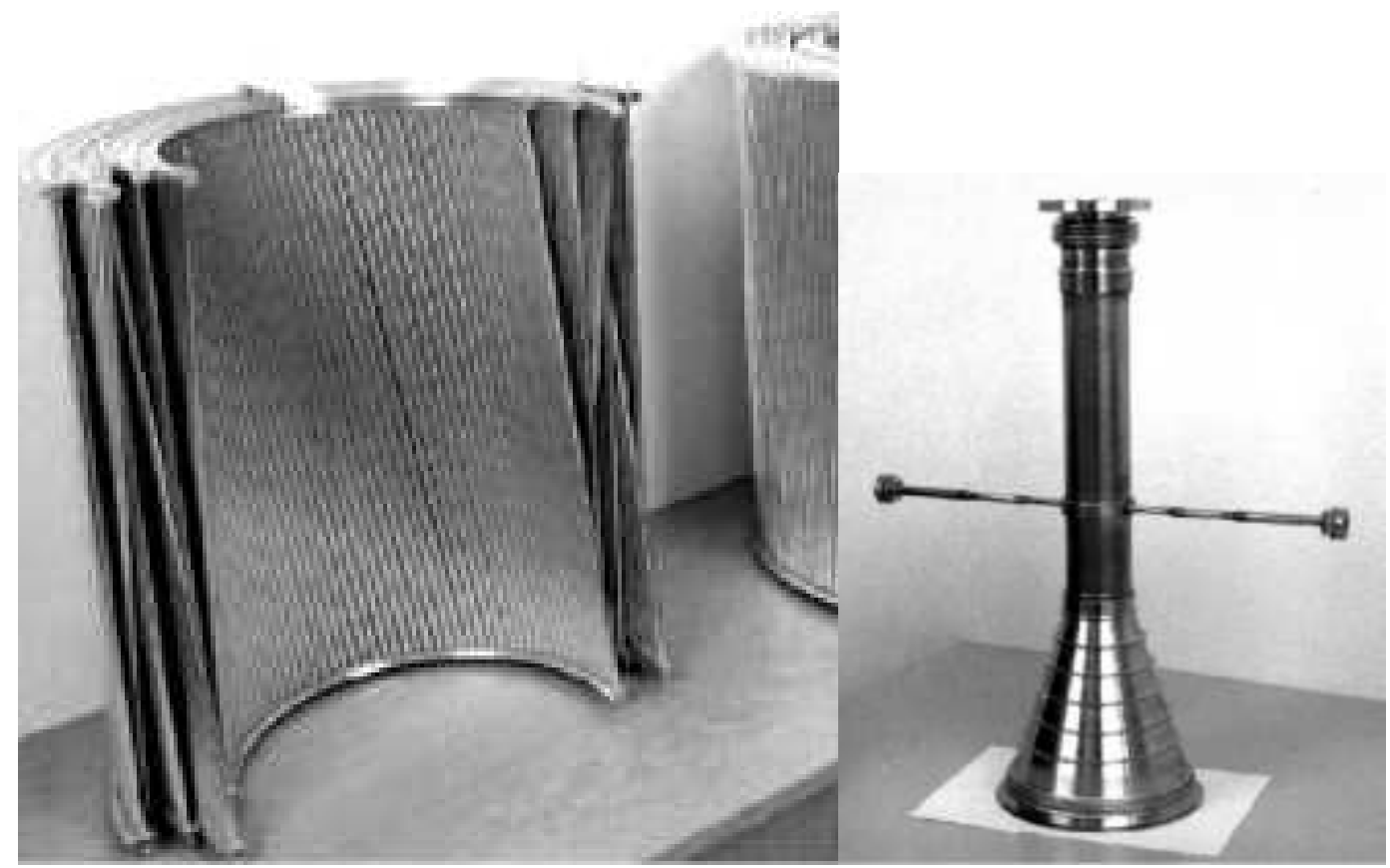

Fig. 11. (Left) Drift tubes secured in the end-plates. Note the stereo layers interleaved with parallel layers. (Right) Be beam pipe with pellet pipe crossing and forward cone.

The MDC consists of 1738 drift tubes, so called straws, arranged in 17 cylindrical layers. The diameter of the straws in the 5 innermost layers is $4 \mathrm{~mm}$, 
$6 \mathrm{~mm}$ in the next 6 layers and $8 \mathrm{~mm}$ in the outer 6 layers. The straws are made of a thin $(25 \mu \mathrm{m})$ mylar foil coated with $0.1 \mu \mathrm{m}$ aluminum on the inner side only. In the center of each straw there is a $20 \mu \mathrm{m}$ diameter sensing wire made of gold plated tungsten (W(Re)), stretched with a tension of $40 \mathrm{~g}$. The wires are aligned with a precision of $\pm 20 \mu \mathrm{m}$.

This design was chosen in order to cope with the expected high particle flux allowing a maximum deposited energy of approximately $70 \mathrm{MeV} / \mathrm{mm} / \mathrm{s}$ for the most exposed straws at the inner part of the chamber.

The layers are located between radii of 41 and $203 \mathrm{~mm}$. The straws in nine layers are parallel to the beam axis (z-axis) and the other eight layers have small skew angles $\left(6^{\circ}-9^{\circ}\right)$ with respect to the z-axis. These stereo layers form a hyperboloidal shape.

Due to the forward cone (Fig. 11), the straws in the five inner layers are positioned unsymmetrically with respect to the pellet pipe. The straws in each layer are inter - spaced by small gaps in order to prevent the mechanical deformation by neighboring tubes.

The MDC is fitted inside a cylindrical cover made of $1 \mathrm{~mm} \mathrm{Al-Be}$ and is placed inside the solenoid (Fig. 10).

The straws in each (half) layer are mounted between $\approx 5 \mathrm{~mm}$ thick Al-Be endplates. The layers are assembled around the Be beam pipe (Fig. 11) and the attached pipe for the pellets. The beam pipe has a diameter of $60 \mathrm{~mm}$ and a wall thickness of $1.2 \mathrm{~mm}$.

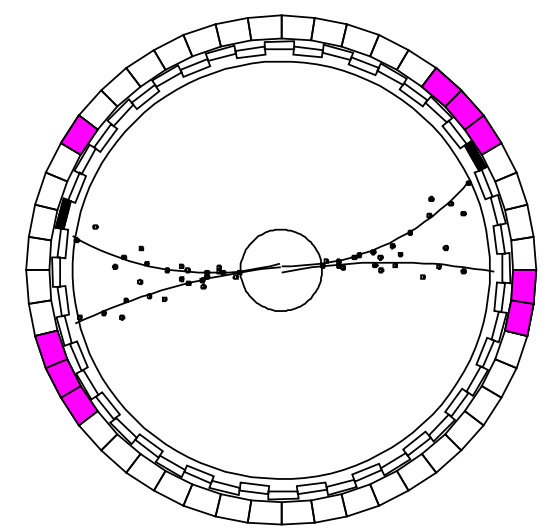

Fig. 12. Tracks in the MDC as seen in the event display of a $\eta \rightarrow e^{+} e^{-} e^{+} e^{-}$decay candidate.

Fig. 12 shows an event display of an $\eta \rightarrow e^{+} e^{-} e^{+} e^{-}$decay candidate. The lines represent the tracks reconstructed in the MDC from the pattern recognition program. The apparent spread of points is due to hits in the stereo layers from forward/backward going tracks. The shaded areas in the outermost rings gives the projection of the hit PSB central elements and the hit SEC crystals (the 
size of the crystals and the radial position of the front faces are not to scale).

\subsubsection{The Plastic Scintillator Barrel - (PSB)}

The PSB is located inside the SCS and surrounds the MDC. It provides fast signals for the first level trigger logic and, together with the mini drift chamber and the CsI calorimeter, it is employed for charged particle identification by the $\Delta E-p$ and $\Delta E-E$ methods and serves as a veto for $\gamma$ identification.

The performance of the PSB has been studied using proton-proton elastic scattering events. Fig. 13 (left plot) shows the result of a Monte Carlo simulation of the angular dependence on the energy deposited in the PSB. Fig. 13 (right plots) shows typical experimental spectra after a correction for nonuniform signal response has been applied.
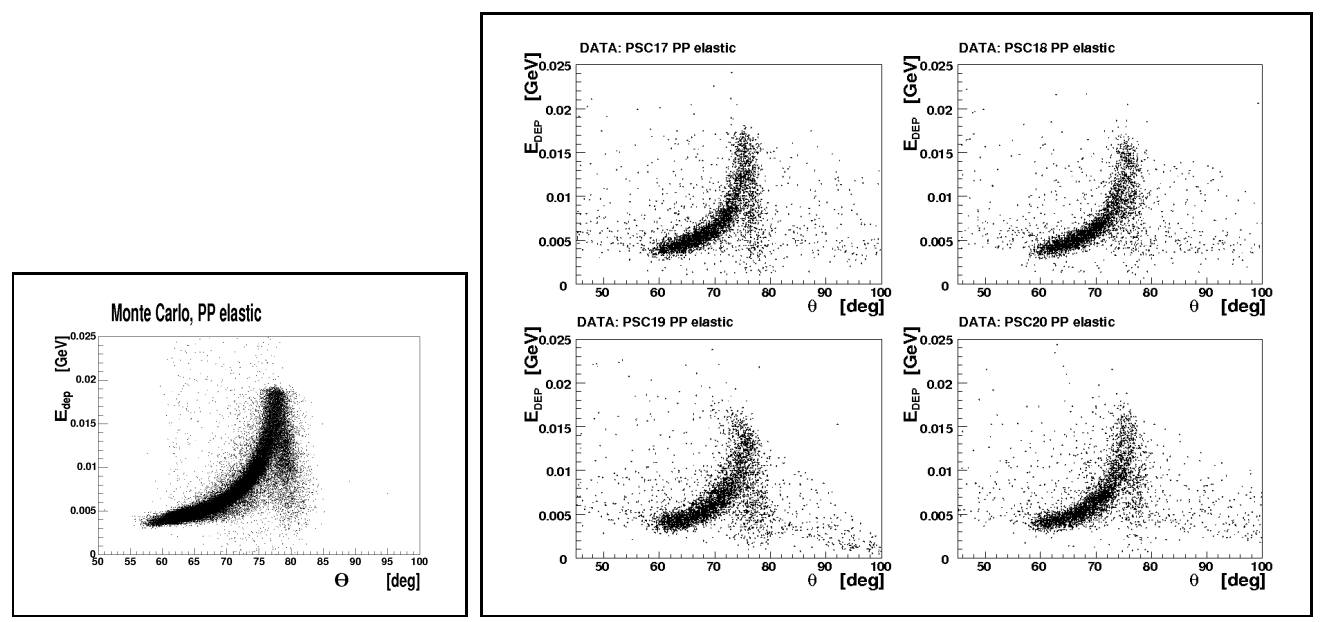

Fig. 13. (Left) Angular dependence of the deposited energy in the PSB for simulated elastically scattered protons. The energy deposition increases with increasing polar angle (corresponding to a decrease of the kinetic energy of the proton), until particles begin to stop in the plastic scintillator material (at around $\theta=77^{\circ}$ ). (Right) Experimental spectra corrected for light attenuation for four of the PSB central elements.

In the initial experiments the momentum and energy resolution allowed reasonable discrimination between pions and protons, which is illustrated in Fig. 14. For high energy charged particles also SEC information is available and can be used for the identification.

The PSB consists of a cylindrical part and two end caps and contains in total 146 elements shaped as strips of $8 \mathrm{~mm}$ thickness. In the cylindrical part there are $48(+2)$ elements of $550 \mathrm{~mm}$ length and $38 \mathrm{~mm}$ width, forming 2 layers with a small (on average $6 \mathrm{~mm}$ ) overlap between neighboring elements to avoid that particles pass without registration. The end caps with an outer diameter of approximately $42 \mathrm{~cm}$ in the backward and $51 \mathrm{~cm}$ in the forward part contain 


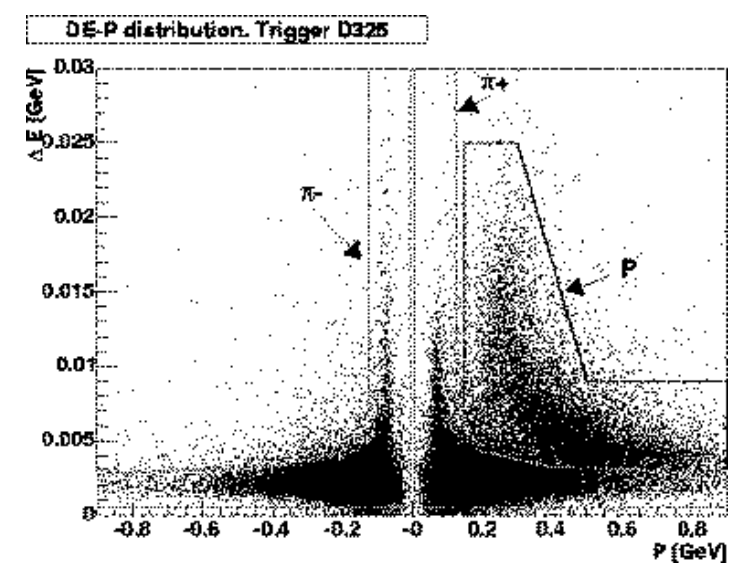

Fig. 14. Example of particle identification in the central detector for a raw data sample collected at $1360 \mathrm{MeV}$. Energy deposited in the PSB is given as a function of signed momentum from the MDC. The regions for protons and pions are marked.

48 cake-piece shaped elements each. The front end cap is flat while the rear cap forms a conical surface. Both end caps have a central hole for the beam pipe $(19 \mathrm{~cm}$ diameter in the forward and $12 \mathrm{~cm}$ diameter in the backward part). One sector of the PSB is shown in Fig. 15.

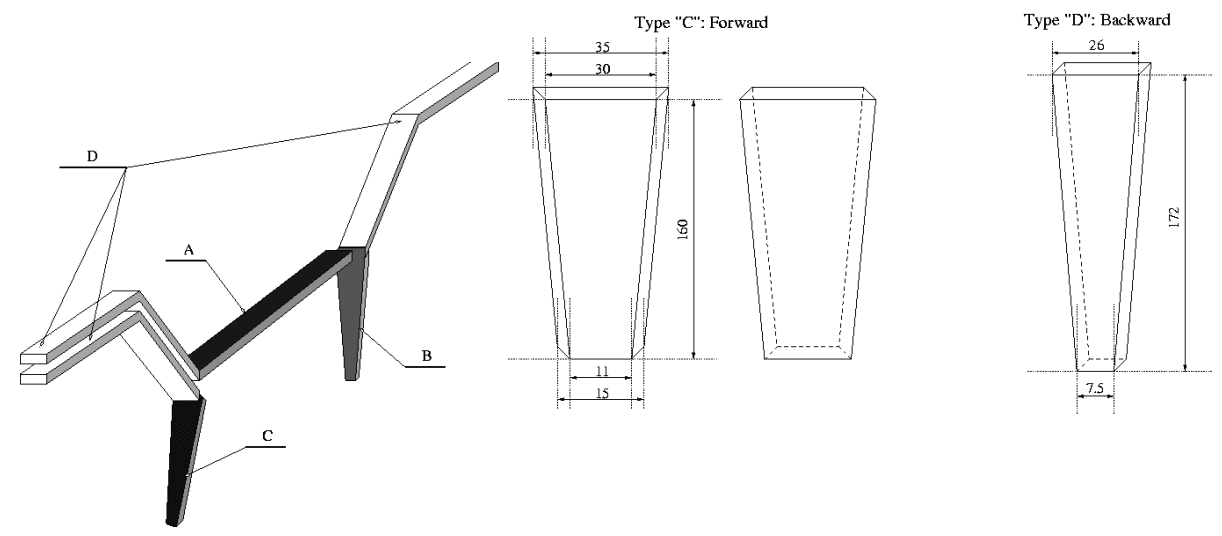

Fig. 15. (Left) Layout of one section of the PSB detector. A denotes the rectangular counters of the barrel wall and $\mathbf{B}$ and $\mathbf{C}$ are trapezoidal elements in the forward and in the backward caps respectively. $\mathbf{D}$ are bent light guides. (Middle) Two shapes of the trapezoidal forward elements with dimensions marked in mm. (Right) Shape and dimensions in mm of the trapezoidal backward element.

Each scintillator is glued to an acrylic light guide coupled to the photomultiplier tube (PMT). The PMTs are placed outside of the iron yoke to shield them from the magnetic field. For this purpose, approximately $50 \mathrm{~cm}$ long light guides are used.

\subsubsection{The Scintillator Electromagnetic Calorimeter - (SEC)}

The CD calorimeter SEC is able to measure photons, electrons and positrons with energies up to $800 \mathrm{MeV}$. The energy threshold for detection of photons 


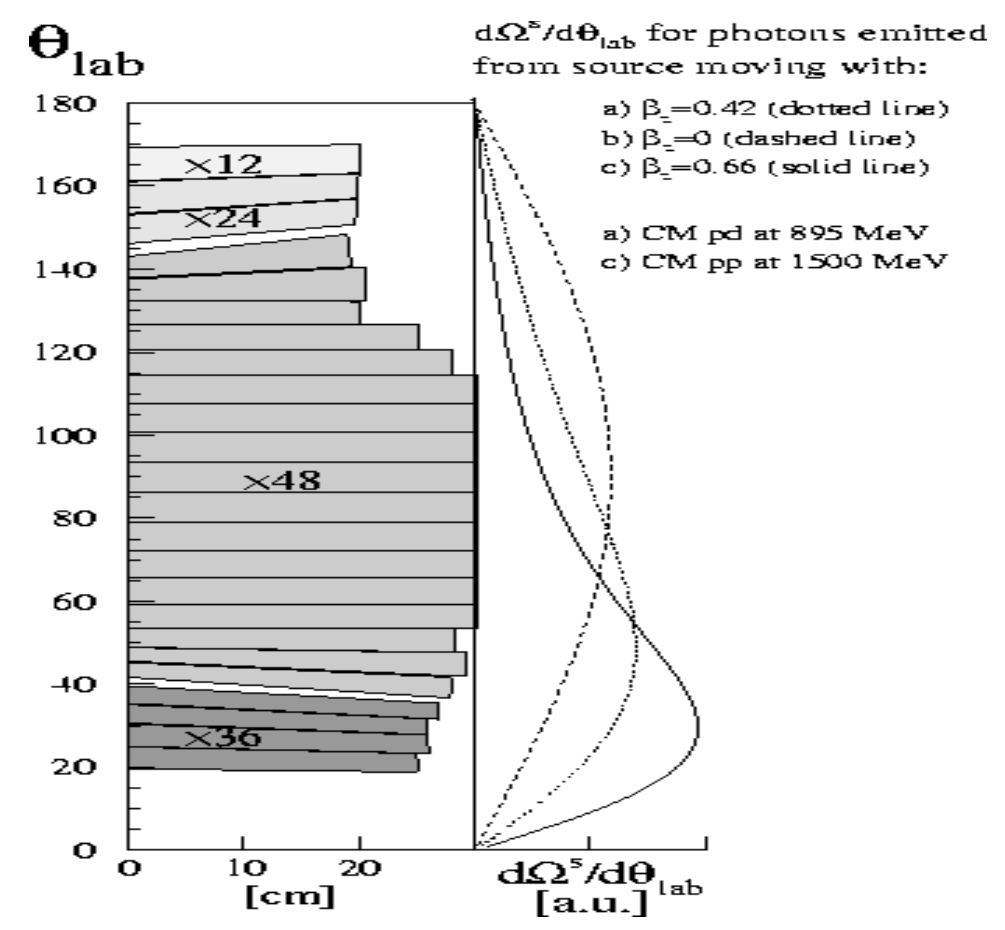

Fig. 16. The angular coverage of the SEC. The CM system solid angle $\left(d \Omega^{s} / d \theta_{L A B}\right)$ vs. the LAB scattering angle $\left(\theta_{L A B}\right)$ is shown for $p p$ and $p d$ interactions at $1500 \mathrm{MeV}$ and $895 \mathrm{MeV}$.

is about $2 \mathrm{MeV}$. The SEC consists of 1012 sodium-doped CsI scintillating crystals placed between the superconducting solenoid and the iron yoke. The scattering angles covered by the SEC are between $20^{\circ}$ and $169^{\circ}$.

The crystals are shaped as truncated pyramids and are placed in 24 layers along the beam (Fig.s 2 and [17). The length of the crystals varies from $30 \mathrm{~cm}$ (16.2 radiation lengths) in the central part to $25 \mathrm{~cm}$ in the forward and $20 \mathrm{~cm}$ in the backward part. Fig. 16 shows the angular coverage together with the thickness of the SEC. As a measure of the anticipated photon fluxes, the center of mass (CM) system solid angle vs. the laboratory (LAB) scattering angle is shown for some experimental conditions at WASA.

The forward part consists of 4 layers with 36 elements each. It covers scattering angles from nearly $20^{\circ}$ to $36^{\circ}$. The central part consists of 17 layers each having 48 elements, and covers scattering angles from $36^{\circ}$ to $150^{\circ}$. The backward part consists of three layers. Two layers have 24 elements and the layer closest to the CELSIUS beam pipe has only 12 elements. The small spaces between the forward-central and central-backward parts are occupied by PSB light guides and mechanical support for the solenoid (back end only). To prevent photons and fast particles to escape undetected, these spaces (and the neighboring layers of crystals) are not pointing exactly towards the interaction region (Fig. 16). The calorimeter covers nearly $360^{\circ}$ in azimuthal angle. Holes for the pellet pipe $(2+2$ crystals $)$ and for the solenoid chimney ( 4 crystals) are 


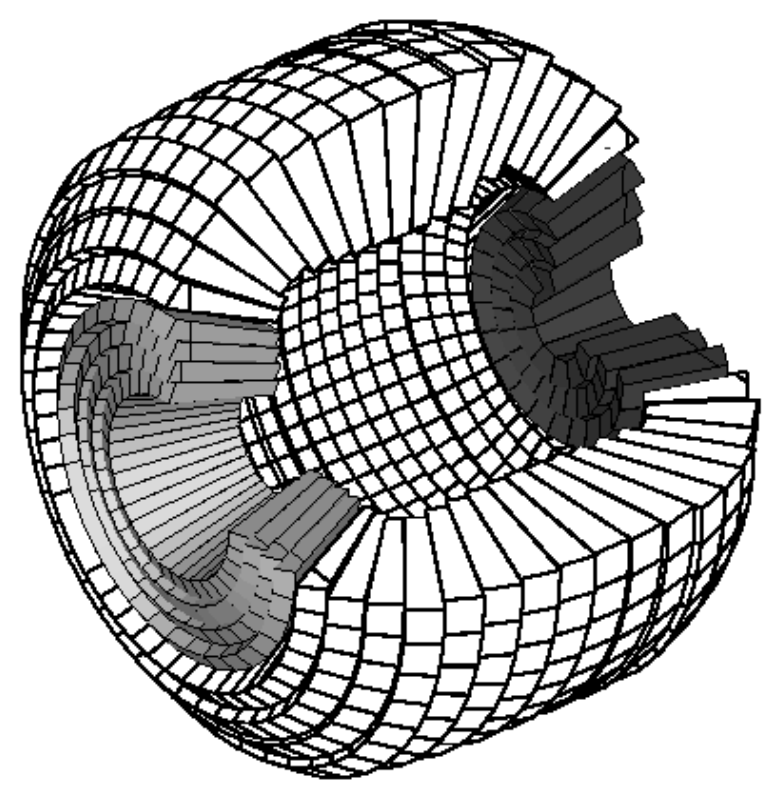

Fig. 17. Schematic view of the SEC. It consists of the forward part (shadowed area to the left), the central part (not shadowed area in the middle) and the backward part (shadowed area to the right). The beam is coming from the right side.

not shown in the figure. Some design parameters of the calorimeter are given in table 4 .

The SEC is composed of sodium-doped CsI scintillating crystals. This type of scintillator material provides a large light yield, has short radiation length and good mechanical properties. $\mathrm{CsI}(\mathrm{Na})$ was chosen instead of the more commonly used $\mathrm{CsI}(\mathrm{Tl})$ scintillators for the following reasons $(19 ; 20)$ :

- Its emission peak at $420 \mathrm{~nm}$ matches well the bi-alkali S11 photocatode of the selected PM tubes, giving good photon statistics and sufficiently fast response.

- Its shorter scintillation decay time is preferable in high-rate applications.

- $\mathrm{CsI}(\mathrm{Na})$ gives much less afterglow than $\mathrm{CsI}(\mathrm{Tl})$.

- $\mathrm{CsI}(\mathrm{Na})$ seems more resistant against radiation damage. When irradiated by a proton beam corresponding to 10 years of operation a test crystal did not show any visible change in its structure. The CsI(Tl) test crystal, on the contrary, lost its transparency.

The crystals are connected by plastic light guides, $120 \mathrm{~mm}$ to $180 \mathrm{~mm}$ long, with the photomultipliers placed on the outside of the iron yoke. In Fig. 18, a fully equipped single calorimeter module consisting of a CsI crystal, a light guide, a PM tube and a high voltage unit, enclosed inside a special housing, is shown. 


\begin{tabular}{lr}
\hline Amount of sensitive material $\left[\mathrm{g} / \mathrm{cm}^{2}\right]$ & 135 \\
$\quad$ [radiation lengths] & $\approx 16$ \\
$\quad$ nuclear interaction length] & $\approx 0.8$ \\
Geometric acceptance: & $96 \%$ \\
$\quad$ polar angle [degrees] & $\approx 20-169$ \\
azimuth angle [degrees] & $\approx 0-360$ \\
Max kinetic energy for stopping & $190 / 400 / 500$ \\
$\quad \pi^{ \pm} /$proton/deuteron & $\approx 5(\mathrm{FWHM})$ \\
Scattering angle resolution $[\mathrm{degrees}]$ & \\
Time resolution [ns] & $5(\mathrm{FWHM})$ \\
charged particles & $\approx 40(\mathrm{FWHM})$ \\
photons & $\approx 3 \%(\mathrm{FWHM})$ \\
Energy resolution & $\approx 8 \%(\mathrm{FWHM})$ \\
charged particles & \\
photons &
\end{tabular}

Table 4

SEC design parameters.

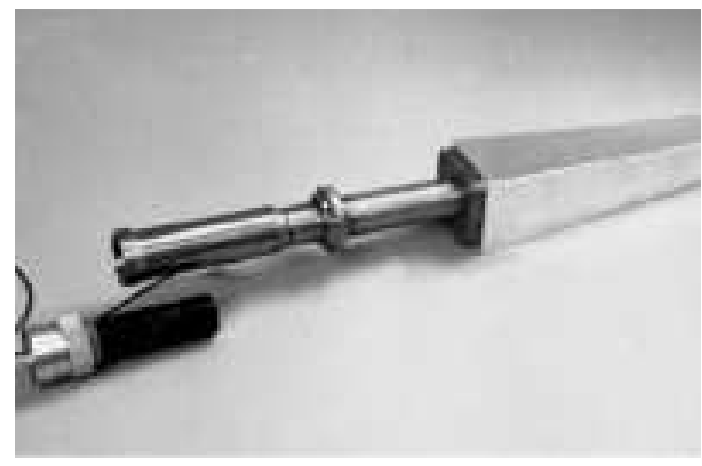

Fig. 18. A fully equipped module with CsI crystal, light guide, PM tube and housing.

The performance of SEC for photon measurements is illustrated in Fig. 19 by data from proton-proton collisions at $1450 \mathrm{MeV}$ beam proton kinetic energy . Events with two protons measured in the FD with a missing mass in the eta region are selected. The plots show the invariant mass of the system of $\gamma$ for events with $2 \gamma$ and $6 \gamma$ detected. The energy resolution in the simulated data (for the $2 \gamma$ case) corresponds to $\sigma_{\mathrm{E}} / \mathrm{E} \approx 0.05 / \sqrt{ } \mathrm{E}(\mathrm{GeV})$. The SEC is described in more detail in the Ph.D. thesis of I. Koch (21). 

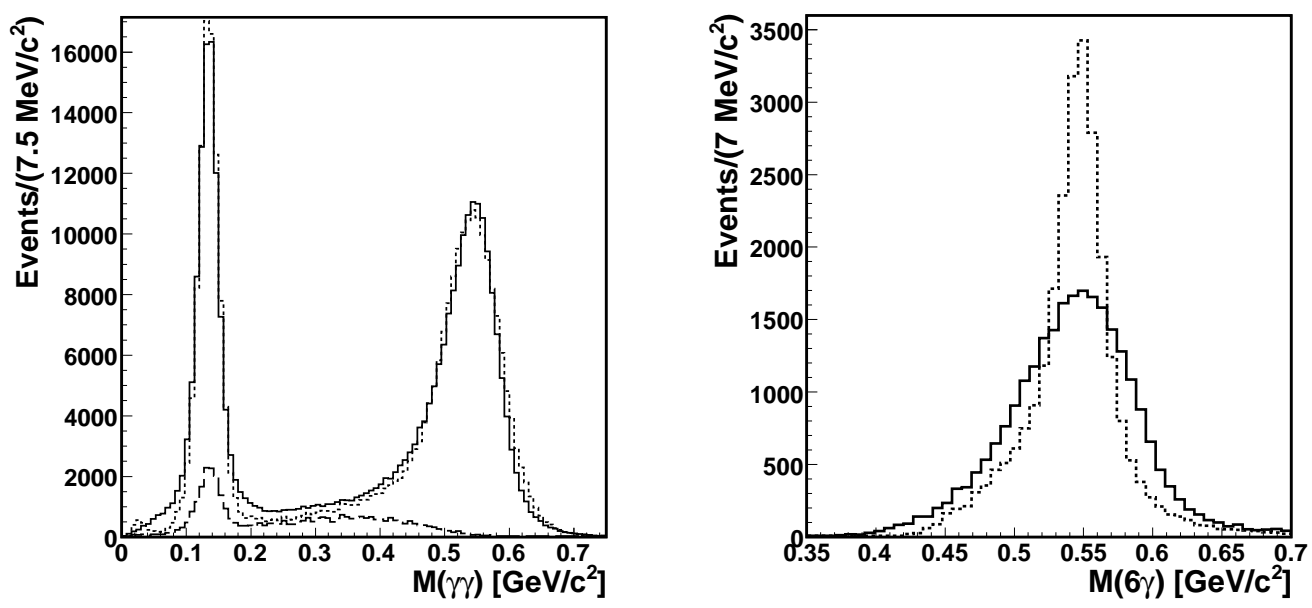

Fig. 19. (Left) Solid line - invariant mass of two $\gamma$. Dotted line - simulated data including the $\eta \rightarrow 2 \gamma$ decay and direct $\pi^{0}$ and $2 \pi^{0}$ production. Dashed line - the contribution from $2 \pi^{0}$ production separately. (Right) Solid line - invariant mass of six $\gamma$ (mainly from $\eta \rightarrow 3 \pi^{0}$ decay), dashed line - after a kinematic fit constrained by that there are three pairs of photons originating from $\pi^{0}$ decays.

\subsection{The Light Pulser System - (LPS)}

The LPS delivers reference light pulses via light fibers to all scintillation counters in order to monitor their gain during the experiment. Since both slow and fast scintillators are used, two types of light sources were designed. A xenon flash tube from Hamamatsu is used for the CsI elements of the calorimeter and three LED-based light sources, of the type used in the PROMICE-WASA setup (22), supply reference pulses to all plastic scintillators. From those four sources the light signals are distributed to individual elements via a network of light fibers. All light sources are monitored for stability, using PIN photodiodes and stable amplifier circuits. The relative resolution of of the light pulse signal amplitude from the plastic scintillators is typically $1.5 \%$. More details about the LPS will be found in ref. (23).

\subsection{Data Acquisition System - (DAQ)}

The DAQ (Fig. 20) handles the signals from the more than 5000 different detector elements of WASA. All signals are discriminated to provide also time information and logic information for use in the trigger. The discriminators for the CD and the FPC were developed within the collaboration while the FD scintillators used commercially available leading edge discriminators. The FPC signals were "time multiplexed" after the discriminators by delaying every 2 nd channel and combining the channels 2 and 2 to reduce the number of digitizing 


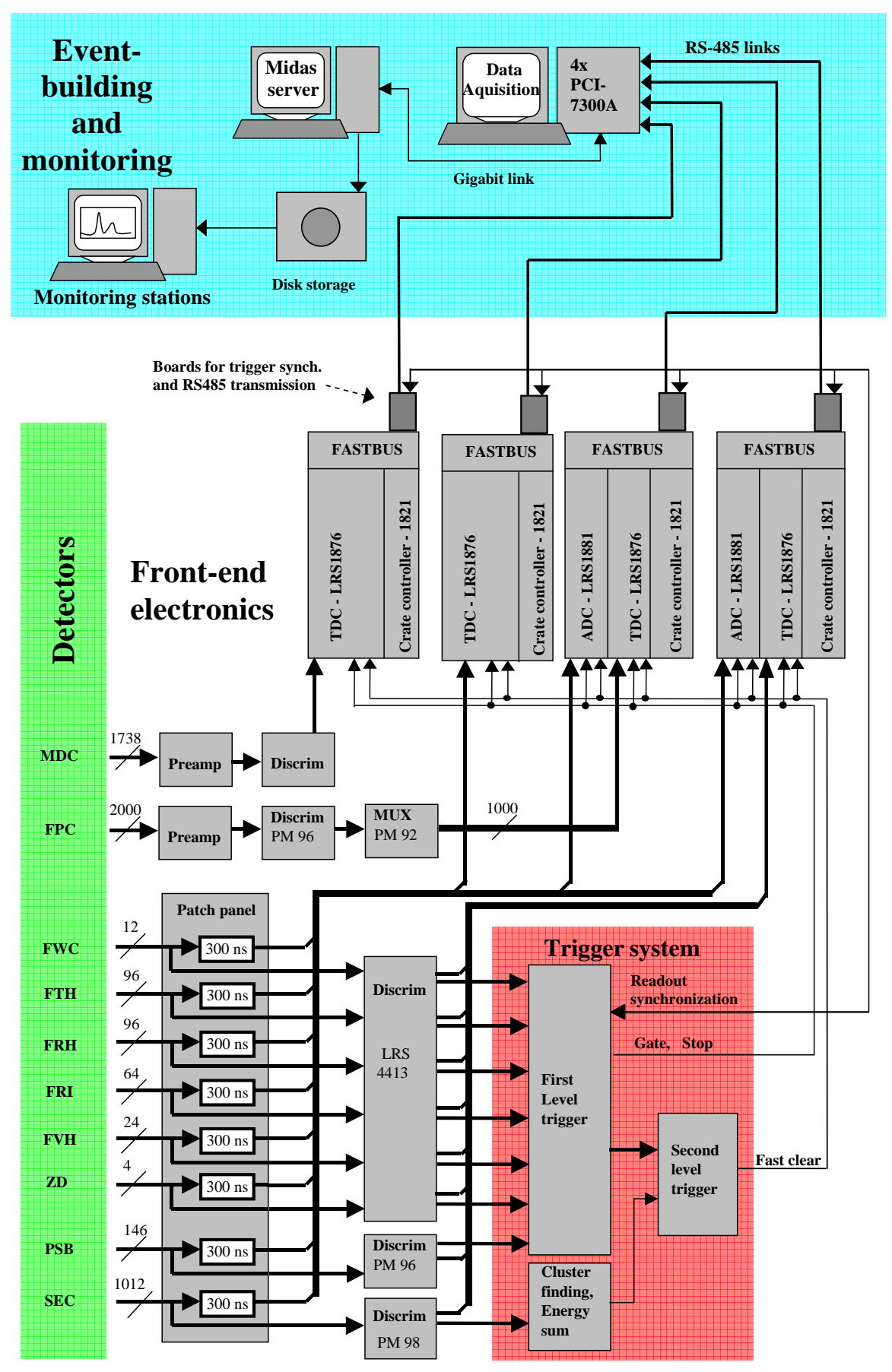

Fig. 20. Structure of the WASA DAQ and trigger system $(24 ; 25)$.

channels.

The trigger system is based on specially developed hardware. The first level trigger uses discriminated signals from the plastic scintillators and generates gates and stops for the QDC's and TDC's. A multiplicity is calculated for each 
detector plane, with clustering of neighbors. Also several consecutive planes, FWC-FTH-FRH, can be required to have matching hits in $\phi$ before calculating the multiplicity. The conditions from the different planes are combined in a 48 input coincidence matrix. In the second level, implemented by using fast clears of the TDC's and QDC's, the signals from the calorimeter are used. The analog PMT signals from the SEC are summed in groups of $4 \times 4$ or $3 \times 4$ and an energy threshold is applied. The logic signals from the groups can be combined with information from the PSB to give information on the number of hits from charged and neutral particles. The analog signal can be further summed to allow a threshold on the total energy in the SEC. An additional method to search for clusters use the discriminated signals from the individual SEC elements. Special hardware search for and count the number of clusters of neighboring elements in the $2 \mathrm{~d}$ SEC matrix. Many parameters of the trigger, like coincidence conditions and delays, could be remotely controlled via an I2C-bus built into the trigger crates.

The readout system is based on digitizing modules in the FASTBUS standard. The charge from the 1500 PMT signals are digitized by 23 LRS1881 2 QDC's and the time information from the MDC, the multiplexed FPC and the discriminated PMT signals, by 47 LRS1876 TDC's. In addition there are three STR200 332 channels scalers for monitoring of trigger rates. (In total over 6000 readout channels). With zero suppression in the QDC's and TDC's this gives an event size of a few kbytes. The modules are distributed over four fastbus crates which are read in parallel. In case of a trigger each LRS1821 crate controller send the data over a proprietary RS485 link to a PCI-7300A interface in the readout PC. An event number for synchronization is added by the link hardware. The PCI-7300A 4 is a 32 bit digital I/O interface with a maximum data rate of 80 Mbytes/s A typical readout time between 250 and $300 \mu \mathrm{s}$ was achieved. The readout PC sends the data from the 4 PCI-7300A over gigabit ethernet to another $\mathrm{PC}$ where the data is written to a disk array and monitoring stations can connect to look at the data. The subevents from the 4 crates are combined offline by the monitoring and analysis programs.

For the ZD internal detector setup, a slower separate data acquisition system was used in parallel to read the Si and Ge detectors (26). A threshold on energy in the Ge detectors provided a typical trigger rate of a few $\mathrm{Hz}$ which also triggered the main DAQ. The main DAQ also recorded the event number from the separate system so that the events could be correlated offline as well as some Ge pulseheight information for cross check.

\footnotetext{
$\overline{2}$ http://www.lecroy.com/lrs/

3 http://www.struck.de/

4 http://www.adlinktech.com/
} 


\subsection{Slow control}

A large number of detector parameters in WASA required monitoring and setup independent of the DAQ. For example the pellet target and solenoid whose operation in addition required a close integration with the operation of CELSIUS. To facilitate this, their control, together with the high voltage for the FD PM tubes and the trigger, was integrated in the same system used to control the TSL accelerators and beam-lines. This runs on VME based VxWorks processors each communicating with a subset of the hardware. All processors are connected together in a dedicated ethernet network with UNIX workstations where graphics interfaces based on Tcl/Tk and SL-GMS Synoptic 5 allows control over the parameters. The parameters are stored in a database on the workstations with local copies on each VME processor of the relevant subset. All parameters are logged at regular intervals as well as changes to the settings.

Also accelerator-cycle dependent quantities like trigger rates, pellet rate and beam current were monitored separately from the DAQ by 3 VME scalers. The MIDAS data acquisition system 6 was used to $\log$ the rates. The web interface of MIDAS could be used to monitor the rates in real time from any computer.

\section{Summary}

The $4 \pi$ detector facility WASA, designed for studies of production and decays of light mesons in proton and deuteron reactions at the CELSIUS storage ring, has been presented. The facility is based on the specially developed pellet internal target system which allowed a detector coverage of close to $4 \pi$ steradians. Large scattering angles are covered by an electromagnetic CsI calorimeter and straw chambers for tracking of charged particles in an solenoidal magnetic field. At forward angles there are straw chambers and a multilayer stack of plastic scintillators. He nuclei escaping in the CELSIUS beam pipe can be detected in a zero-degree spectrometer.

\footnotetext{
5 http://www.sl.com/

6 http://midas.psi.ch/
} 


\section{Acknowledgments}

We gratefully acknowledge the financial support given by the Knut and Alice Wallenberg Foundation, the Swedish Council for Planning and Coordination of Research, the Swedish Research Council, the Göran Gustafsson Foundation, the Swedish Royal Academy of Science, the Swedish Institute, the Swedish Foundation for International Cooperation in Research and Higher Education, the Polish Ministry of Science and Higher Education PBS 7P-P6-2/07, the German BMBF (06HH152, 06HH952, 06TU261, 06TU201, 06TU987), the Russian Foundation for Basic Research (RFBR 02-02-16957), the Russian Academy of Science, the Joint Institute for Nuclear Research in Dubna, and the Japanese Ministry of Education. We also want to thank the technical and administration staff at The Svedberg Laboratory and at the participating institutes.

\section{References}

[1] S. Holm, A. Johansson, S. Kullander, D. Reistad, New Accelerators in Uppsala, Phys. Scripta 34 (1986) 513-532.

[2] M. Bashkanov, et al., Exclusive measurements of $p d \rightarrow^{3}$ Нe $\pi \pi$ : The ABC effect revisited, Phys. Lett. B637 (2006) 223-228.

[3] C. Pauly, et al., The $p p \rightarrow p p \pi \pi \pi$ reaction channels in the threshold region, Phys. Lett. B649 (2007) 122-127.

[4] P. Thörngren Engblom, et al., Anisotropy in the pion angular distribution of the reaction $p p \rightarrow p p \pi^{0}$ at $400 \mathrm{MeV}$, Phys. Rev. C76 (2007) 011602.

[5] M. Bashkanov, et al., Measurement of the slope parameter for the $\eta \rightarrow$ $3 \pi^{0}$ decay in the $p p \rightarrow p p \eta$ reaction, Phys. Rev. C76 (2007) 048201.

[6] C. Bargholtz, et al., Measurement of the $\eta \rightarrow \pi^{+} \pi^{-} e^{+} e^{-}$decay branching ratio, Phys. Lett. B644 (2007) 299-303.

[7] M. Berlowski, et al., Measurement of eta meson decays into leptonantilepton pairs, Phys. Rev. D 77 (3) (2008) 032004.

[8] B. Trostell, Vacuum injection of hydrogen microsphere beams, Nucl. Instrum. Meth. A362 (1995) 41-52.

[9] C. Ekström, et al., Hydrogen pellet targets for circulating particle beams, Nucl. Instrum. Meth. A371 (1996) 572-574.

[10] H. Calén, et al., Detector setup for a storage ring with an internal target, Nucl. Instrum. Meth. A379 (1996) 57-75.

[11] J. Dyring, Detailed Studies of the Reaction $p p \rightarrow p p \eta$ using a Straw Chamber Tracking Device, Ph.D. thesis, Uppsala University (1997).

[12] M. Dahmen, et al., A three layer circular scintillator hodoscope, Nucl. Instrum. Meth. A348 (1994) 97-104. 
[13] C. Pauly, Light Meson Production in pp Reactions at CELSIUS/WASA above $\eta$ Threshold, Ph.D. thesis, Hamburg University (2006).

[14] C. Pauly, L. Demirörs, Y. Stark, S. Vorrath, W. Scobel, A scintillator hodoscope for experiments at proton storage rings, Nucl. Instrum. Meth. A547 (2005) 294-301.

[15] C. Bargholtz, et al., Tagging of eta mesons using the $p d \rightarrow^{3}$ He eta reaction near threshold, Instrum. Exp. Tech. 49 (2006) 461-467.

[16] R. J. M. Y. Ruber, et al., An Ultra-thin-walled solenoid for the CELSIUS/WASA experiments, Nucl. Instrum. Meth. A503 (2003) 431-444.

[17] R. J. M. Y. Ruber, An Ultra-thin-walled Superconducting Solenoid for Meson-decay Physics, Ph.D. thesis, Uppsala University (1999).

[18] M. Jacewicz, Measurements of the Reaction $p p \rightarrow p p \pi^{+} \pi^{-} \pi^{0}$ with CELSIUS/WASA at $1.36 \mathrm{GeV}$, Ph.D. thesis, Uppsala University (2004).

[19] R. J. M. Y. Ruber, Radiation hardness test of CsI crystals for WASA, WASA Report 2/90 (1990).

[20] U. Schuberth, The Reaction $p p \rightarrow p p \eta$ Close to Threshold, Ph.D. thesis, Uppsala University (1995).

[21] I. Koch, Measurement of $2 \pi^{0}$ and $3 \pi^{0}$ Production in Proton-Proton Collisions at a Center of Mass Energy of $2.465 \mathrm{GeV}$, Ph.D. thesis, Uppsala University (2004).

[22] J. Zabierowski, High power LED pulser for gain monitoring in a multicounter experiment, Nucl. Instrum. Meth. A338 (1994) 577-581.

[23] J. Zabierowski, in preparation.

[24] K. Fransson, The trigger system of the CELSIUS/WASA detector, Phys. Scripta T99 (2002) 176-182.

[25] P. Marciniewski, Fast Digital Trigger Systems for Experiments in High Energy Physics, Ph.D. thesis, Uppsala University (2001).

[26] B. Hovander, A Flexible IBM PC based data acquisition system for nuclear physics experiments, Nucl. Instrum. Meth. A325 (1993) 266-270. 